\title{
Le processus d'orientation scolaire au Maroc
}

Une analyse sociologique

A Sociological Analysis of the Careers Advice Process in Moroccan Schools

Un análisis sociológico del proceso de orientación escolar en Marruecos

\section{Ali Boulahcen}

\section{OpenEdition}

\section{Journals}

Édition électronique

URL : http://journals.openedition.org/ries/1427

DOI : 10.4000/ries. 1427

ISSN : 2261-4265

\section{Éditeur}

Centre international d'études pédagogiques

Édition imprimée

Date de publication : 1 avril 2005

Pagination : 25-34

ISSN : 1254-4590

Référence électronique

Ali Boulahcen, « Le processus d'orientation scolaire au Maroc », Revue internationale d'éducation de Sèvres [En ligne], 38 | avril 2005, mis en ligne le 01 avril 2008, consulté le 21 décembre 2020. URL http://journals.openedition.org/ries/1427 ; DOI : https://doi.org/10.4000/ries.1427 


\title{
Le processus d'orientation scolaire au Maroc
}

\section{Une analyse sociologique}

\begin{abstract}
Ali Boulahcen
L'objet de cette étude consiste à mener une analyse du processus d'orientation scolaire au Maroc par le biais d'une analyse des procédures et des pratiques d'orientation scolaire mises en œuvre au «point de bifurcation» qui se situe à la sortie du collège : la $3^{\mathrm{e}}$ année secondaire collégial ( $3^{\mathrm{e}} \mathrm{ASC}$ ). Je me limite à ce seul et unique palier d'orientation du système éducatif où la carte scolaire, concrétisant la volonté socio-politique de l'État, fixe le taux d'orientation à $47 \%$ en moyenne de l'ensemble des élèves de la $3^{e}$ ASC. Quel que soit l'établissement scolaire - et donc que ce soit un collège à population plus bourgeoise, plus populaire ou typiquement rurale - la même règle s'applique de façon stricte. Quant aux 53\% restants, ils doivent soit redoubler ou tripler la classe s'ils en ont le droit, soit passer les tests d'accès à la formation professionnelle pour obtenir un diplôme de qualification, soit enfin entrer directement et sans qualification sur le marché du travail.

Les matériaux théoriques mais aussi pratiques mis en œuvre pour analyser le processus d'orientation scolaire à la sortie du collège et notamment au niveau de la classe de $3^{\mathrm{e}}$ ASC sont de deux types : d'une part, une enquête qualitative par entretien avec douze conseillers d'orientation ${ }^{1}$ pour rapporter leurs différentes pratiques; d'autre part, l'observation directe du déroulement de trois "conseils d'orientation» dans chacun de ces établissements (bourgeois, populaire et rural) afin de mieux cerner la différence qui existe entre les divers discours et la réalité.
\end{abstract}

\section{LES ATTRIBUTIONS DES CONSEILLERS EN ORIENTATION}

\section{Le rôle du conseiller en orientation}

Théoriquement, le rôle du conseiller en orientation est de bien distinguer les élèves capables de poursuivre avec succès leurs études secondaires ou

1. Quatre par districts (villas, quartiers et campagne), deux hommes et deux femmes à chaque fois, dont un(e) débutant(e) et l'autre expérimenté (e). 
professionnelles et ceux qui ne le sont pas. Cependant, avant d'arriver à cette étape de sélection, il commence par donner des informations aux élèves sur les différentes sections. Il est ainsi censé connaître et décrire toutes les "chaînes de mobilité» scolaires, tous les dispositifs institutionnels qui permettent de réussir des études ultérieures.

Pour une conseillère d'un collège de la périphérie pauvre de la ville, «le conseiller en orientation est un agent, un expert en la matière, dans la mesure où il intervient chaque fois qu'il y a un problème qui se pose à l'élève au niveau administratif, éducatif, etc.». Ainsi le conseiller fait de son mieux pour aider, dans un premier temps, l'élève à dépasser ses difficultés, à résoudre ses problèmes... C'est en tenant compte de tout cela qu'il parvient à aider les jeunes élèves à s'exprimer puis à choisir leur voie d'orientation. Un autre conseiller auprès d'un collège de quartier confirme que le rôle du conseiller en orientation est très important parce qu'il ne se contente pas d'orienter les élèves mais les informe et leur apprend à choisir eux-mêmes leur branche d'orientation.

La tâche des conseillers en orientation en secteur scolaire rural est encore plus ardue. En effet, si les élèves citadins sont à tout le moins concernés par l'orientation scolaire, les élèves des collèges de campagne sont de plus en plus coupés de cette réalité alors qu'ils ont un besoin plus important d'information et d'orientation. À l'évidence les réponses fournies par les orienteurs marocains témoignent d'une volonté de bien faire, mais ils sont confrontés à la lourdeur de structures administratives centralisées et autoritaristes, héritage de l'administration coloniale française.

\section{Une fonction efficace?}

Tous les conseillers, sans exception, répondent, chacun à leur façon, qu'ils ne sont pas satisfaits de ce qu'ils font en matière d'orientation au niveau des collèges. Je rapporte ici quelques-unes de leurs réactions significatives.

Les conseillers contestent vigoureusement les procédures d'orientation mises en œuvre jusqu'à présent. Ils seraient d'accord pour que tout change à ce sujet. «Je ne suis pas du tout satisfait de ce que je fais en orientation. Je crois que je ne suis pas en train de faire ce que je devrais faire, c'est ce qui me pèse».

Une conseillère en collège de villas exprime un rejet catégorique des pratiques d'orientation en cours. "Je ne suis pas satisfaite de ce que je fais en orientation au collège parce que ce que je fais ici, ce n'est pas de l'orientation. L'orientation doit être continue, c'est-à-dire qu'elle doit s'étaler, du moins au collège, sur les trois années pour accompagner le développement mental et personnel mais aussi professionnel de l'élève. En effet, parallèlement à son développement personnel, il y aura chez l'élève une ouverture professionnelle qu'il faudra stimuler. Maintenant, ce que je fais, c'est de l'information sur les seules filières scolaires qui existent et l'élève choisit sa branche sans avoir profité d'une orientation continue». 


\section{La collaboration avec les parents et les professeurs}

La relation socio-pédagogique entre les conseillers en orientation et les parents d'élèves ainsi que les professeurs semble être problématique dans presque tous les milieux étudiés. Un conseiller en collège de villas reconnaît qu' «avec les parents, quelquefois, il y a des réunions mais c'est rare. Avec les professeurs, j'ai eu, une fois, une seule rencontre [...] Nous avons eu une discussion à propos de l'évaluation des [...] épreuves de l'examen du premier trimestre. Nous avons aussi discuté d'autres difficultés et ils ont pris note. [...] Ça ne s'est jamais répété car on a eu des problèmes avec le directeur ». De son côté, une jeune conseillère en veut davantage aux parents d'élèves qu'aux professeurs. "Les parents, je ne travaille pas avec eux. J'ai essayé à maintes reprises de les inviter, de les convoquer à des réunions, à des conseils de classes, mais ils ne viennent jamais. Les professeurs, je suis toujours en contact avec eux».

En réalité, parents, professeurs et conseillers ont à se concerter, non pas seulement sur ce qui concerne l'orientation scolaire des élèves, par exemple sur les critères qu'il faut mettre en ouvre pour orienter un élève vers telle ou telle branche, mais aussi sur les contenus d'enseignement.

La collaboration avec les parents et les professeurs est également difficile pour les conseillers en collège de secteur rural. Et ils ont, eux aussi, du mal à s’inscrire dans une certaine logique de concertation. Un conseiller expérimenté le dit ainsi : "j'ai demandé au directeur de réunir quelques parents d'élèves. Le directeur m'a adressé au président de l'association locale des parents. Le président m'a dit qu'il ne pouvait réunir que deux ou trois parents. J'ai vu que ce nombre était insuffisant, j'ai abandonné ». Il faut ajouter que même si des parents viennent lui demander des renseignements, ils ne le trouvent pas car il ne dispose pas d'un bureau personnel au sein de l'établissement. L'initiative d'organiser une réunion peut même être mal perçue : «je crois que ça va créer des problèmes. On va se demander : «Que fait ce conseiller en orientation?»».

\section{LES DÉCISIONS D'ORIENTATION}

\section{Les facteurs déterminants}

Dans le souci de bien cerner la diversité des facteurs qui entrent en jeu au moment du processus d'orientation scolaire, j'ai interrogé les conseillers sur l'importance du sexe de l'élève, de son âge, de ses notes et de ses choix d'une part, et d'autre part sur celle de la demande familiale ainsi que sur la prise en compte des observations des professeurs. Les réponses paraissent varier d'un milieu à l'autre mais aussi, au sein d'un même milieu donné, selon les cas particuliers qui se présentent. 
Ainsi, malgré la spécificité du milieu favorisé où exerce l'un des conseillers interrogés, qui compte déjà quelques années d'expérience, presque toutes les variables prises en considération semblent poser des problèmes. Ainsi, d'après lui, les collèges de villas sont actuellement presque tous mixtes et le taux de réussite des filles dépasse parfois celui des garçons "peut-être parce que la famille bourgeoise marocaine s'émancipe de plus en plus». Mais aussi le fait que certains parents essaient d'imposer leurs choix sans tenir compte des résultats de leurs enfants.

Par ailleurs, le phénomène des cours particuliers fausse l'évaluation. En effet, certains professeurs se permettent de donner les notes les plus élevées aux élèves auxquels ils donnent des cours particuliers. "Un élève qui a 5/20 au premier trimestre, puis $6 / 20$ au deuxième trimestre se retrouve avec 15 ou 16/20 au troisième trimestre. [...]. On a des exemples flagrants. Dernièrement, dans un conseil d'orientation dans un collège, les notes que donnait un professeur de mathématiques m'ont surpris. Les mathématiques ont le coefficient 5 , c'est-àdire un coefficient élevé. Il a donné des 18 et 19/20 à la majorité de ses élèves, ce qui fait que dans sa classe, dix-sept élèves ont été orientés en sciences et uniquement deux élèves en lettres. Une fois, [...] j'ai attiré l'attention des professeurs et même du directeur qui a protesté contre moi sous prétexte que je portais atteinte à l'honneur de ses professeurs en plein conseil». Ce chef d'établissement, comme beaucoup d'autres, essaie de créer un malentendu entre le conseiller et les professeurs pour que le conseiller reste l'élément «indésirable » bien qu'il ait de bonnes intentions et souhaite collaborer avec tout le monde.

Quelques conseillers travaillant dans des établissements ruraux parviennent à s'affirmer sur le terrain. C'est le cas du jeune conseiller qui souligne clairement la part de toutes les variables déterminant l'orientation des élèves de la $3^{\circ} \mathrm{ASC}$ dans son secteur. "Je constate que les filles sont encore loin des branches techniques et même de la branche scientifique. Les garçons souhaitent s'orienter vers la branche technique et agricole mais la carte scolaire n'accorde que $5 \%$ pour les deux». Mais il ne faut pas oublier que, dans les collèges ruraux, les filles représentent généralement moins de $10 \%$ du total des élèves. Cette situation reste typiquement liée à la ruralité : d'abord parce que l'établissement est éloigné des villages, ensuite parce que, par tradition, les parents préfèrent garder leurs filles près d'eux, et enfin en raison des représentations sociales.

\section{Les critères d'orientation}

Dans le cadre de l'enquête, les conseillers en orientation des élèves en milieu rural se limitent en général à deux réponses. Un jeune conseiller affirme qu' «il n’y a ni test ni rien. Il n'y a que la note, les $47 \%$ imposés par la carte scolaire et le choix de l'élève». Un deuxième ajoute qu' «en tant que membre du conseil d'orientation, [il] ne [s]e base que sur la moyenne». 
De leur côté, même s'ils se contentent, eux aussi, de la note afin d'orienter les élèves, quelques conseillers intervenant dans les collèges de quartiers prennent en outre en compte l'avis des professeurs et aspirent à une certaine orientation continue; d'autres tiennent davantage en second lieu au choix de l'élève et ils revendiquent enfin le changement radical des critères d'orientation mis en ouvre jusqu'à présent. Les propos tenus par une jeune conseillère confirme ces tendances : "La note et l'avis des professeurs, c'est tout ce dont je dispose. Si je faisais de l'orientation continue, il y aurait d'autres critères». En effet, l'orientation continue permettrait à l'élève la connaissance de soi et de l'environnement scolaire. Ainsi, il ferait, en principe, un bon choix à la fin «Si je prenais, dit-elle, en considération la personnalité de l'élève, ce qu'il aimerait, ce qu'il pourrait faire, etc., normalement, il devrait faire le bon choix. Mais avec ce que j'ai, avec ce que je fais maintenant, je ne me base que sur la note, l'avis des professeurs, c'est tout. Je n'ai pas de fiches techniques de suivi et je n'ai pas de papier pour les imprimer moi-même».

La seconde tendance des conseillers qui travaillent dans les établissements populaires est rapportée par quelqu'un qui compte déjà plusieurs années d'expérience : «j'aimerais bien que la parole soit donnée à l'élève. Il s'avère juste que les élèves doivent avoir leur part de parole, ils doivent être représentés. Que l'élève participe au conseil d'orientation reste un impératif. On décide de son avenir sans qu'il le sache. Je vois que c'est injuste». Pour ce qui est du reste des critères, «je dois dire qu'il y a la barrière de la planification : la Carte scolaire. Je suis entre le marteau de la Carte scolaire et l'enclume du choix de l'élève qui doit être exaucé. Je ne sais vraiment quoi ou qui satisfaire».

\section{L'OBSERVATION DES CONSEILS D'ORIENTATION}

Dans le cadre de ma procédure d'enquête, j'ai assisté à trois conseils d'orientation de trois classes de $3^{\circ} \mathrm{ASC}$ dans trois collèges de villas, de quartiers et de campagne. Mon objectif était d'en décrire l'organisation et le déroulement.

C'est le chef de l'établissement qui préside le conseil d'orientation scolaire : il consulte successivement par ordre alphabétique les livrets scolaires des élèves, annonce dans un premier temps le nom et le prénom des élèves, puis sa moyenne générale annuelle, échange avec les autres membres du conseil sur les décisions à prendre et enfin, porte et signe lui-même sur le bulletin de l'élève l'observation finale du conseil.

À côté du directeur du collège, les professeurs de toutes les matières d'enseignement, en possession de leur carnet de notes mensuelles, trimestrielles et annuelles, suivent attentivement toutes les étapes rituelles consacrées à l'examen de chaque cas d'élève. Ils prennent note à chaque fois de la moyenne générale de la classe, puis de celles obtenues par chaque élève dans les diffé- 
rentes matières et finalement de la décision finale prise par le conseil. Il est fort rare de les voir comparer leurs notes au moment du conseil et ils ne le font que si la discussion s'attarde sur un cas particulier.

Quant au conseiller en orientation, il est en charge des dossiers scolaires des élèves à l'intérieur desquels se trouve leur fiche de vœux, qui lui ont été remises bien avant la tenue des conseils. À son tour, il porte sur chaque dossier scolaire les différentes moyennes générales de la classe et de chaque élève ainsi que la décision finale, suivie de la mention accordée à l'élève par le conseil d'orientation. Le conseiller ne se réfère au cursus antérieur marqué sur le dossier scolaire que s'il y a divergence entre les différents assesseurs sur la décision à prendre vis-à-vis d'un élève.

En somme, on ne discute que rarement cas par cas. Il s'avère ainsi que tout est joué à l'avance car, avant de commencer le conseil d'orientation et avant même de citer le premier élève en tête de la liste, le chef d'établissement annonce à tous la moyenne générale de toutes les classes de $3^{\circ} \mathrm{ASC}$ du collège et celle qui constituera le seuil de réussite, sans tenir aucun compte des différentes filières d'orientation. Seul est pris en compte le taux de passage en lycées d'enseignement général et technique, fixé à $47 \%$ en moyenne par la Carte scolaire et imposé par le Service central du Ministère de l'Éducation Nationale à tous les collèges du royaume. Il est donc très rare que la concertation de l'ensemble des membres ou une quelconque contestation émanant de l'un ou de l'autre des participants vienne perturber le climat social chaud mais muet du conseil d'orientation. C'est aussi pour cette raison que ce conseil, qui est supposé se passer en une heure pour chaque classe, ne se déroule en réalité qu'en moins d'une demi-heure et cela pour presque toutes les classes dans tous les collèges.

Pour ce qui est des fonctions du conseil d'orientation, je ne vois en lui qu'un instrument par le biais duquel l'institution tente de réaliser, parmi la population scolarisée, une certaine répartition, en deux catégories. En ce sens le conseil d'orientation vient concrétiser l'objectif de la politique éducative qui, n'ayant pas les moyens financiers de scolariser toute la population marocaine avant, durant et après la période de la scolarité obligatoire (elle va de 6 à 15 ans) consiste à inciter les jeunes élèves inscrits en $3^{\circ} \mathrm{ASC}$ à quitter en masse $(53 \%$ en moyenne) le système éducatif pour rentrer dans le système productif. Ces jeunes passant d'ailleurs rarement par des Centres de formation professionnelle qui leur permettraient d'obtenir éventuellement une qualification. Mais ces Centres, d'ailleurs en nombre suffisant, recrutent sur tests; ce qui fait que la majorité des jeunes entrent sur le marché du travail sans qualification professionnelle. C'est ainsi que les exigences de la vie active distinguent les travailleurs manuels, qui sont de l'ordre de $80 \%$, des intellectuels, qui représentent $20 \%$.

La véritable fonction du conseil d'orientation n'est donc pas d'aider les enfants à choisir la voie leur permettant de développer leurs facultés mentales, 
mais essentiellement de préparer les uns pour le marché du travail «une main d'œuvre plus ou moins qualifiée» et les autres pour devenir « une élite intellectuelle» capable d'assurer la gestion socio-économique mais aussi administrative et politique du pays.

Par ailleurs, au lieu de contribuer à compenser et à réduire les différences sociales existant entre les élèves (car il lui est impossible de les éliminer), le conseil d'orientation scolaire ne fait que les aggraver, par la contrainte de ce taux de passage fixé à $47 \%$ en moyenne pour tous les collèges. Ainsi peut-on dire qu'il engendre des handicaps au lieu de les combattre. Le redoublement en est le premier exemple. L'orientation scolaire contribue ainsi à l'inégalité des chances au sein de l'enseignement public, car il n'est mis en place que pour répondre à la demande du système économique. Il n'est alors qu'une instance de régulation qui permet le placement des individus parmi les différents niveaux de l'échelle socio-économique.

Cette inégalité est déjà institutionnellement marquée en amont de ce premier palier d'orientation à la sortie du collège où, par le jeu des redoublements d'abord et de l'échec ensuite, se différencient les carrières scolaires et professionnelles. La différence entre les trois types de collèges (villas, quartiers et campagnes), l'inadéquation des contenus des programmes d'enseignement et enfin la fixation du taux d'orientation vers l'enseignement général et technique à $47 \%$ en moyenne accentuent encore plus les différences entre ces élèves, qui ont pourtant la chance d'être scolarisés et, par là-même, influent sur leurs trajectoires scolaires et professionnelles ultérieures. Les différences de performances en classe, fortement liées à l'appartenance sociale mais engendrées en fait par l'école, sont en effet manifestes bien avant ce palier d'orientation scolaire.

La même division existe aussi très clairement au niveau de l'enseignement secondaire principalement entre les filières de l'enseignement général qui débouchent sur les études académiques longues de l'enseignement supérieur, (études conduisant à une double domination, culturelle et sociale), et les filières techniques et surtout professionnelles qui ouvrent sur des formations courtes dans des classes de transition débouchant sur des filières de techniciens.

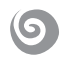

Nous avons pu remarquer à travers cette analyse qu'au fond, il n'y a pas de véritable processus d'orientation scolaire, mais qu'il n'y a qu'un processus sommaire dans le cadre duquel on décide, en quelques secondes, du sort de l'élève en se basant uniquement sur sa valeur scolaire traduite par une note chiffrée dont tout le monde doute de la réelle valeur. C'est dire que l'institution scolaire marocaine se base, à ce niveau du moins, sur des critères de 
sélection et non d'orientation. Les méthodes «intuitives» et «irrationnelles » de l'orientation sont ainsi critiquées sévèrement au Maroc.

Réduite à des conseils ponctuels, l'orientation scolaire reste "l'arme » institutionnelle tranchante entre les mains du système éducatif marocain qui s'en sert pour légitimer l'inégalité des chances qu'il traduit au terme de la scolarité dite obligatoire (jusqu'à l'âge de 15 ans) en classe de $3^{\circ} \mathrm{ASC}$, dernière année de l'enseignement secondaire collégial. L’orientation est donc le mécanisme institutionnel qui permet de persuader tout élève non orienté de se résigner au statut hérité de sa catégorie sociale. C'est pourquoi, d'ailleurs, cette instance d'orientation ne garde, à ce niveau, qu'un sens unique : elle est généralement scolaire pour ceux qui ont les moyens de suivre la voie royale d'abord et elle n'est même pas professionnelle pour les autres qui manquent de tout, sauf de leur détermination et de leur acharnement pour tenter de réussir leur intégration socioprofessionnelle ultérieure.

Il s'avère donc nécessaire de réfléchir à une organisation scientifique de l'orientation scolaire capable de réguler les flux d'élèves vers les différentes filières d'enseignement de façon raisonnable et juste mais aussi de créer les passerelles permettant, si besoin est, de changer d'orientation en cours de scolarité.

Enfin, il serait utile que les élèves puissent être conduits à s'approprier le sens et la portée de leur responsabilité et de leur engagement personnel notamment au sein du conseil d'orientation. Il faudrait faire en sorte que par leurs projets scolaires et professionnels, les élèves arrivent à se construire un projet de vie. Pour ce faire, n'est-il pas temps de conquérir tout d'abord en amont cette introuvable relation triangulaire Éducation/Orientation/Formation avant de se précipiter ensuite à la recherche en aval de la relation binaire Formation/Emploi? Ce n'est pas dans une école injuste que l'élève démuni pourra s'épanouir et, ainsi se stabiliser.

\section{BIBLIOGRAPHIE}

BERTHELOT J.-M. (1987) : «De la terminale aux études post-bac : itinéraires et logique d'orientation». Revue Française de Pédagogie, No 81, pp. 515.

BOULAHCEN A. (1991) : L'inégalité des chances devant l'enseignement au Maroc. Université de Nancy 2, 143 p.

BOULAHCEN A. (2002) : Sociologie de l'Éducation. Les systèmes éducatifs en France et au Maroc. Étude comparative. Casablanca : Afrique-Orient, $237 \mathrm{p}$. 
BOULAHCEN A., BARCELONA MONITORING (collectif) (2004) : Suivi de l'accord de libre-échange. Dimension de l'Éducation et de la Formation. Rabat : Éditions de la Fondation Friedrich Ebert, 264 p.

BOURDIEU P. et PASSERON J.-Cl. (1970) : La reproduction. Éléments pour une théorie d'enseignement. Paris : Minuit, 270 p.

BRIAND J.-P. et CHAPOULIE J.-M. (1992) : Les collèges du peuple. E.N.S. Fontenoy-Saint-Cloud. Paris : I.N.R.P. - C.N.R.S., 544 p.

CHARLOT B. (1992) : «Rapport au savoir et rapport à l'école dans deux collèges de banlieue». Sociétés contemporaines, $\mathrm{N}^{\circ} 11$, pp. 119-147.

DEROUET J.-L. (1988) : «Désaccords et arrangements dans les collèges. Éléments pour une sociologie des établissements scolaires». Revue Française de Pédagogie, $\mathrm{N}^{\circ}$ 83, pp. 5-21.

DUBAR C. (1991) : La socialisation : Construction des identités sociales et professionnelles. Paris : A. Colin, 278 p.

DURU-BELLAT M. (1988) : Le fonctionnement de l'orientation : genèse des inégalités sociales à l'école. Paris : Lauzanne, Delachaux et Niestlé, 199 p.

ECKERT H. (1993) : L'orientation professionnelle en Allemagne et en France. Paris : L'Harmattan, 310 p.

IBAAQUIL L. (1987) : Mobilité sociale et passage par l'école au Maroc. Université de Paris 5 : Thèse d'État, 1444 p.

MORACCHINI Ch. (1992) : Système éducatif et espaces fragiles : les collèges dans les montagnes d'Auvergne. Clermont-Ferrand : C.E.R.A.M.A.C., 255 p.

PASSERON J.-C. (1989) : «Biographie, flux, itinéraires, trajectoires». Revue Française de sociologie, $\mathrm{N}^{\circ}$ XXXI, pp. 3-22.

SALAMI J. (1985) : Crise de l'enseignement et reproduction sociale au Maroc. Casablanca : Maghrébines, 225 p.

THÉLOT Cl. (1982) : Tel père, tel fils : position sociale et origine familiale. Paris : Dunod, 249 p.

TERRAIL J.-P. (1992) : «Parents, filles et garçons face à l'enjeu scolaire». Éducation et Formation, $N^{\circ} 30$. 
